\title{
Les enjeux relationnels d'une fidélisation de papier : les magazines de marque, entre pratiques et représentations
}

\section{Caroline de Montety}

\section{(2) OpenEdition} Journals

Édition électronique

URL : http://journals.openedition.org/communicationorganisation/3245

DOI : 10.4000/communicationorganisation.3245

ISSN : 1775-3546

\section{Éditeur}

Presses universitaires de Bordeaux

\section{Édition imprimée}

Date de publication : 1 juin 2005

Pagination : 98-110

ISSN : 1168-5549

\section{Référence électronique}

Caroline de Montety, «Les enjeux relationnels d'une fidélisation de papier : les magazines de marque, entre pratiques et représentations », Communication et organisation [En ligne], 27 | 2005, mis en ligne le 19 décembre 2012, consulté le 19 avril 2019. URL : http://journals.openedition.org/ communicationorganisation/3245 ; DOI : 10.4000/communicationorganisation.3245

Ce document a été généré automatiquement le 19 avril 2019

(c) Presses universitaires de Bordeaux 


\title{
Les enjeux relationnels d'une
} fidélisation de papier : les magazines de marque, entre pratiques et représentations

\author{
Caroline de Montety
}

1 Les opérations et outils de fidélisation se développent et représentent un pourcentage croissant du budget de communication des entreprises. Aux mailings, succèdent des opérations de marketing téléphonique, diffusions de newsletters, propositions d'adhésion à un club, envois de SMS... Les pratiques du marketing, intimement liées à nos vies quotidiennes et banalisées, sont valorisées par les entreprises, enseignées dans les universités, relatées dans les médias. Généralement analysées pour être vantées par les professionnels du marketing ou mises en cause par leurs détracteurs, ces pratiques ne sont de fait que rarement interrogées en dehors de leur dimension idéologique.

2 L'exemple des magazines de marque permettra d'observer certains enjeux relationnels, destinés à produire de la fidélité. Le contrat de lecture, revendiqué par les professionnels comme outil participant à leur élaboration, sera exploré pour comprendre sur quelles représentations de la communication il s'appuie pour fidéliser et ce qu'il advient de ce contrat dans les mains des lecteurs. Ce travail d'analyse permettra en conclusion d'aborder la question des modèles sémiotiques adoptés et diffusés par les entreprises. Sans prétendre à une théorie communicationnelle générale des pratiques marketing, j'espère par cette présentation contribuera à éclairer un de ses aspects: celui de la confrontation entre représentations de la communication mobilisées par les entreprises et pratiques des consommateurs. 


\section{Le développement du marketing relationnel et ses enjeux}

3 Le développement du marketing relationnel semble lié à une évolution du marketing induite par plusieurs dynamiques :

4 - La prise de conscience d'une relation au client avec la mise à jour des principes du «marketing des services ».

5 - La comparaison des coûts d'acquisition d'un nouveau client avec ceux de leur pérennisation, bien moins élevés.

6 - L'engouement pour les technologies disponibles et le coût de leur utilisation, la conjugaison d'une promotion efficace de ces «nouvelles » pratiques, avec le succès du one to one et des ouvrages qui l'ont promu, et la mise en cause de l'efficacité publicitaire traditionnelle.

7 - La montée des marques et leur dynamique d'extension dans les différents champs sociaux, leur valorisation au regard de l'adhésion qu'elles suscitent chez leurs consommateurs.

8 Selon G. Marion, l'expression «marketing relationnel » peut être évoquée dans de multiples acceptions sans que l'on puisse évoquer un changement de paradigme. L'observation des différentes pratiques du marketing liées à cette expression permet de distinguer deux cas de figures :

9 - Une intensification des interactions grâce aux nouvelles techniques pour gérer des bases de données finement segmentées.

10 - Une intensification des liens qui unissent un consommateur à une entreprise en comparant ce lien avec un lien social ou affectif plus fort.

11 Dans le premier cas, nous pouvons évoquer les opérations telles que marketing one to one, $C R M$, qui font appel à l'individualisation des prestations et des interactions avec les clients. Dans le second cas, nous retrouvons tous les usages métaphoriques des relations sociales avec le souhait de favoriser une relation dans ce qu'elle a d'impliquant et de durable. La relation marchande dépasserait le simple cadre de la transaction pour revêtir un visage plus humain, s'appuierait sur des éléments affectifs et engagerait le consommateur. C'est cette acception du marketing relationnel que je souhaite retenir afin de montrer les enjeux majeurs présents dans les termes de marketing relationnel:

12 - Le déplacement du concept de cible à celui de fidèle.

13 - Le déplacement du principe de contrôle de la transaction au contrôle de la relation : le contrôle d'un lien social qui irait au-delà du lien marchand.

14 La promesse d'une fidélité est une promesse de constance, d'engagement, d'habitude, de croyance. Elle est très désirable pour des entreprises exposées aux risques de la concurrence, de l'indifférence, de l'inconstance, alors qu'elles désirent une fréquence des achats dans le temps et la valorisation de ses clients dans la durée. Comment rendre compatible la visée économique de l'entreprise avec l'idée de fidélité? Les marques semblent au cœur du processus car en tant que médiations, moteurs sémiotiques porteurs de cohérence, elles mettent en avant des valeurs, inscrivent des transactions dans un environnement symbolique: créent une relation. Ce déplacement métaphorique est 
facilité par leur caractère anthropomorphique, évoqué dans les termes d'identité de marque, personnalité de la marque, ADN de la marque, qui masquent les conditions de leur élaboration.

Nous avons choisi de nous intéresser à l'un des multiples moyens de fidélisation: les magazines de marque, nommés aussi consumer magazines. Quels sont les enjeux d'une relation médiatique qui devrait inciter à faire d'un lecteur un fidèle d'une marque?

\section{Les magazines de marque : formes médiatiques et stratégie communicationnelle}

Ils sont plus de cent cinquante en France que nous définissons ainsi, faute d'acception officielle: publications périodiques, accessoires d'une activité commerciale pour une marque donnée et ayant adopté tout ou partie des caractéristiques formelles de la presse magazine. Leur point commun est d'être édité à l'instigation d'entreprises qui souhaitent renforcer l'achat de leur marque (marque-enseigne, marque-produit ou service...) directement (stimulation du trafic), ou indirectement (renforcement de l'image de marque). Cette désignation permet de juxtaposer deux instances médiatrices de nature différente: l'une destinée à un lecteur, l'autre à un consommateur. L'expression est emblématique d'un double statut informationnel et publicitaire. Si ces magazines de marque existent depuis la fin du XIX ${ }^{e}$ siècle, leur développement récent illustre le déploiement des marques dans des terrains où l'on ne les attend pas, leur investissement d'autres supports que les médias traditionnels encombrés, coûteux. Le genre magazine, appréhendé dans sa valeur informative et sa valeur de support, associé à l'idée d'objectivité et de crédibilité, notions habituellement associées au journalisme, présente de multiples atouts pour les entreprises. Sa forme accessible et plaisante, ses informations mêlant quotidien, modes de vie et consommations, en font un prescripteur objectif et légitime. Ainsi, en éditant leur propre magazine, les entreprises réalisent un investissement apte à conférer à leur discours un statut qui le rende audible et crédible. Si la forme magazine est désirable pour valoriser les discours sur les marques, la plasticité de ces dernières permet une adaptation à tous les types de supports. Leur nature sémiotique favorise la construction de réseaux de signification, leur statut économique et communicationnel leur permet de basculer d'un champ à l'autre. peuvent en espérer divers bénéfices, d'ordre cognitifs, affectifs et conatifs :

18 - une présence à l'esprit chez le consommateur,

19 - l'apprentissage de la marque, de ses valeurs et produits,

20 - une reconnaissance,

$21 \cdot$ une préférence,

22 - une incitation à passer à l'action grâce à la connaissance des "événements ", nouveautés, politiques promotionnelles de la marque, ...bénéfices qui seraient provoqués par :

23 - la pénétration, non intrusive, de la marque dans le foyer,

24 - un moment de satisfaction, procuré par la lecture, accru par le don (fréquent) du magazine, avec l'appréciation d'un traitement préférentiel, voire le sentiment d'appartenance à une communauté privilégiée, 
$$
\begin{aligned}
& \text { marque éditrice et l'établissement d'une relation pérenne et rentable pour l'entreprise : } \\
& \text { la certitude d'achats récurrents, voire la captation exclusive des achats dans la catégorie } \\
& \text { de biens et services promus, l'élargissement du panier moyen du consommateur. }
\end{aligned}
$$

- l'accompagnement grâce au conseil,

- la possibilité d'une consommation différée et symbolique...

Ces bénéfices auraient pour conséquence un attachement durable du consommateur à la marque éditrice et l'établissement d'une relation pérenne et rentable pour l'entreprise : de biens et services promus, l'élargissement du panier moyen du consommateur.

Si cette représentation est commune aux agences et entreprises, on peut se demander comment elle est mise en œuvre et sur quelles conceptions de la relation de lecture elle repose.

\section{Glissements et métamorphoses}

29 Grâce à une recherche sémio-pragmatique réalisée sur un large corpus de magazines de marques (une soixantaine) puis sur un corpus restreint et homogène de publications dédiées à l'habitat ${ }^{1}$, j'ai analysé la rhétorique de ces supports. L'analyse énonciative permet d'observer la relation proposée au consommateur dans le processus de fidélisation. J'ai repris la conception traditionnelle de la pragmatique, héritée de $\mathrm{K}$. Bühler, selon laquelle la communication peut être comparée à un univers à trois pôles réunissant l'énonciateur, le destinataire et le monde dont ils s'entretiennent. Cette conception fixiste m'a permis d'observer le discours dans les magazines de marque et de mettre en lumière les glissements au sein de chacun de ces pôles. L'énonciateur se présente comme une entité brouillée dans chacun des magazines: on constate une superposition entre le monde dont on parle, l'univers de la marque, et l'énonciateur, métaphore de la marque. L'énonciateur est en effet hybride ou flou : à la fois représentant de l'entreprise, relais de ses activités marchandes ou institutionnelles, entité médiatique apparemment indépendante... Si dans certains articles et magazines, les énonciations se fondent, dans d'autres les voix alternent, tandis que dans certains les énonciations se masquent. Dans tous les cas, les oscillations entre une énonciation culturelle et une énonciation marchande, le brouillage dans ces supports hybrides, semblent destinés à faire oublier au lecteur, même fugacement, qu'il est une cible.

30 Ce même travail de brouillage et ces mêmes glissements sont présents dans la figure du destinataire, interpellé à la fois comme consommateur, habitant, citoyen, individu intégré dans des rapports sociaux et familiaux, individu pris dans des modes et des mouvements collectifs, à la fois acteur et être agi par le magazine. Il est invité à consommer le magazine et le magasin, à entrer dans un dialogue entre représentations collectives et ses propres représentations.

31 Le monde construit est aussi un monde hybride traversé par des logiques publicitaires et journalistiques. Si l'intégration des éléments propres à la marque est inégalement habile, tous les magazines de marque utilisent les codes du magazine pour évoquer l'univers de consommation. Le discours se trouve ainsi naturalisé. La relation au monde proposée par l'énonciateur valorise la marque mais en gardant la manière de dire du magazine :

32 - Une relation didactique pour une marque positionnée comme savante, avec un savoir souvent pratique et accessible, proposé à un destinataire désireux de conseils et de mises en œuvre. La relation est, grâce au média, « complémentaire », didactique. 
33 - Une relation de spectacle avec une scénarisation des objets, dont certains sont des produits de la marque, naturellement mis en avant pour favoriser un plaisir esthétique et créer des ambiances.

34 Une relation de proximité, très présente dans les magazines de marque, avec une valorisation des personnalisations possibles et le glissement du collectif au personnel grâce à des effets de projection :

35 - On observe de nombreuses formes dialogiques suggérant des interpellations: interrogations, exclamations, jeux sur les pronoms (avec des passages du nous au vous).

- La mise en avant d'une intégration dans un système de normes collectives déterminantes des comportements individuels : saisons, fêtes etc.

37 - La mise en scène de personnages saisis "sur le vif », dans leur quotidien, renforce la proximité avec la mise en place d'un regard intime sur les choses et les êtres. Il permet de faire entrer le lecteur dans l'intimité d'un monde qui n'est pas le sien mais dont il est intrinsèquement proche, de donner l'illusion d'une absence de médiation.

- Les désirs et particularités du lecteur sont entretenus et reconnus dans la rhétorique de ces publications, grâce à l'évocation de l'adaptation à son propre cas et de solutions particulières suggérant une certaine " personnalisation » : on peut planter dans son parc ou sur son balcon chez Truffaut, on peut faire évoluer ses aménagements selon la configuration de sa famille et les étapes de la vie chez Room, adapter sa décoration chez Maisons en vie, etc.

39 Les glissements révèlent un implicite fort : le passage du voir à l'avoir, de la lecture à la consommation. Le recours au magasin est le moyen d'y accéder. Ce basculement proposé au lecteur n'est jamais explicité, il est au mieux suggéré grâce à des marqueurs placés aux endroits stratégiques (la «Une ») et parfois aussi par la référence directe à la marque (avec la mise en avant de sa signalétique, de ses produits...). C'est en tout cas au lecteur qu'est laissée la reconstitution de ce lien, masquant le caractère intentionnel du propos. Ceci favorise la participation d'un lecteur devenu "co-énonciateur ». La relation de proximité s'en trouve considérablement enrichie: en s'impliquant dans la lecture, le lecteur est en mesure de s'approprier le discours de la marque et de la consommer symboliquement.

40 Dans ce système communicationnel, à la fois, prédéterminé et souple, hybride et cohérent, le discours médiatique est construit de façon à ce qu'il soit efficace pour les marques : ostensiblement journalistique et disculpé de son intention publicitaire.

\section{Le contrat de lecture au cœur de la relation construite dans le magazine}

41 La lecture est utilisée comme un moyen de fidéliser le consommateur en suscitant son adhésion à l'univers symbolique bâti autour de la marque. Les bricolages énonciatifs que nous avons évoqués permettent de bâtir un mode énonciatif métaphorique des effets souhaités sur le destinataire grâce à la lecture qui rappelle le contrat de lecture tel qu'il a été développé par E. Veron et E. Fouquier. Cet outil d'analyse, qui se réfère à la pragmatique, établit un lien entre représentations et pratiques et à ce titre permet de penser la relation, de la formaliser en tant que résultat d'un message élaboré dans un contexte médiatique. Cet outil d'analyse de la relation a été modélisé à l'origine dans le 
cadre du médiaplanning pour distinguer des positionnements de titre de presse. Le terme de marque n'est pas cité, mais il s'agit bien là de mettre au jour des identités de marque (les titres de presse étant aussi des marques), grâce à l'analyse des régularités et à la comparaison d'un titre à l'autre. magazines de marque, même si pour eux ce contrat a des définitions différentes de celle d'E. Véron et si les usages de l'expression varient selon les agences. Le contrat est dit de lecture mais est fondé sur la stratégie globale du texte vis-à-vis de la cible. Généralement, il détermine le contenu médiatique, est une condition de la fidélité de la lecture et un outil pour favoriser la fidélité à la marque. Le passage d'un type de fidélité à l'autre est favorisé par la reconnaissance de la marque dans le texte et la prise en compte de ce que certains nomment le " contrat de marque ». Ainsi, les attributs de la marque visibles dans le magazine doivent être cohérents avec les autres productions de l'entreprise : valeurs de marque, signalétique etc. La stabilité de la marque doit s'accompagner, sauf changement de "formule ", d'une stabilité du texte, fidèle d'un numéro à l'autre aux choix de rubriques, de types d'information, aux choix iconiques... Le contrat de lecture des agences est conçu comme un engagement fondé sur une double représentation : celle de ce qui est intéressant pour un lecteur, selon les caractéristiques de la cible, et une représentation de ce qui est souhaitable pour l'entreprise éditrice et sa ou ses marques. Dans les magazines de marque, le contrat reposerait sur l'intégration de cette « double contrainte ", sur un discours médiatique promouvant la marque éditrice, ses prestations, ses valeurs, sur un mode discret mais récurrent et cohérent. produits mais à les produire. Cette transformation du statut de l'outil permet de l'utiliser comme méthodologie pour décliner une marque dans un projet éditorial. Il y a ainsi un basculement, comme l'ont constaté Y. Jeanneret et V. Patrin, de l'étude du dispositif 
énonciatif au concept opératoire ${ }^{2}$. Ce basculement semble lié à certaines caractéristiques du modèle: facilement adaptable, peut être parce que la "cuisine» pour l'élaborer n'apparaît pas dans les travaux d'E. Veron et parce qu'il a été exposé en des termes qui n'exigent pas de connaissances théoriques particulières, il réduit et systématise une relation complexe, en la déterminant comme portée à l'intérieur du texte, au point de pouvoir la modéliser. C'est justement cette réduction et systématisation qui peuvent être désirables pour des marketers soucieux de détenir des clés, y compris les clés discursives, pour maîtriser le marché. Le constructivisme de cette approche sémio-pragmatique s'harmonise avec le constructivisme du marketing, inhérent à sa vocation et à son désir de mise en œuvre. La fidélisation semble ici s'inscrire dans la régularité du texte pensé comme cadre structurant, stabilisateur de la relation et comme métaphorique du passage de la lecture à l'achat.

\section{Du lecteur au consommateur fidèle : entre déconstruction et braconnage}

On peut se demander comment le contrat, s'il est envisagé comme outil opératoire, peut susciter de la fidélité dans le cadre des magazines de marque. Même si le déplacement métaphorique construit dans le texte est perçu, cela suffit-il à susciter, dans la réalité, le même déplacement de la lecture à l'adhésion à la marque ? La sélection qu'opère le professionnel ou le chercheur pour élaborer son contrat de lecture est-elle la même que celle qu'opère le lecteur dans son parcours du magazine ? Grâce à l'analyse des magazines du corpus, et en particulier de leurs glissements structurels, j'ai voulu montrer comment ces choix énonciatifs, et les conceptions qu'ils impliquent, étaient manipulés par les lecteurs et se confrontaient à leurs propres représentations. J'ai pour cela confronté une trentaine de lecteurs aux magazines de marque du corpus restreint, sous réserve qu'ils en aient déjà lu un. Cette caractéristique conduit à n'interroger a priori que des personnes non hostiles à ce type de publications. Bien entendu, l'objectif n'étant pas de mesurer l'efficacité des magazines de marque mais bien les représentations qu'ils engagent, je n'ai pas cherché à mesurer leur impact véritable.

La démarche de publication d'un magazine de marque est positivement valorisée, par opposition aux démarches de la publicité intrusive, et grâce au caractère plaisant ou utile du magazine. Le caractère prescripteur du magazine est souligné : la plupart des lecteurs font état de l'utilisation du magazine pour " prendre des idées ", évoquent son pouvoir onirique et suggèrent ainsi son emprise sur les imaginaires dans la mesure où il participe à la circulation des modes, des idées, des représentations. La donnée implicite, à savoir qu'il s'agit ici d'une publication conçue pour favoriser le basculement de lecteur en acheteur, en renforçant son image de la marque et sa venue en magasin, est en général reconstruite par les lecteurs, conscients des glissements du discours. Le travail de fidélisation est même assez finement reconstitué par certains qui envisagent le magazine comme une élaboration à partir des méthodes du marketing: études, diffusion des produits, adaptation aux cibles, segmentation, travail de l'image de marque etc. Dans quelques cas, rares, la déconstruction ne se fait pas et le magazine est appréhendé en tant que tel, sans qu'une différence soit perçue entre cette publication et un magazine de décoration traditionnel. Enfin, certains lecteurs, tout en étant conscients de la dimension publicitaire du magazine, ne sont pas en mesure d'affirmer quelle est la nature de 
l'émetteur du magazine. Il est difficile dans ces deux derniers cas de garder la notion de contrat, la base du contrat étant de pouvoir partager le cadre de l'échange.

Les entretiens soulignent une liberté de lire, de penser et d'agir. De nombreux lecteurs évoquent de multiples pratiques de lecture: feuilletages, jetages, détournements (découpages de recettes, utilisation d'une page comme modèle pour une aquarelle...) mais aussi collection, outil de présélection, «souvenir » de shopping, etc. Ils évoquent aussi la liberté de penser et d'agir : le magazine peut servir des projets formulés ailleurs, ne pas être accompagné d'achat, entraîner un achat dans une autre enseigne.

51 Au-delà de l'aptitude et de l'attitude des cibles à déconstruire sémiotiquement le support, à le replacer dans le contexte économique et énonciatif, à dissocier le plaisir publicitaire de l'achat, les lecteurs mettent en avant la valorisation du magazine à partir de leurs propres désirs. La proposition médiatique s'accorderait ainsi une certaine axiologie de la consommation, fondée sur la valorisation de bénéfices exclusivement ou conjointement pratiques, utopiques, esthétiques, critiques ${ }^{3}$. Les lecteurs se présentent comme des "bricoleurs ", nouant un dialogue muet entre leurs conceptions et leurs envies et ce qui est proposé dans les pages offertes. Les marques apparaissent de ce fait dans leurs magazines comme des opérateurs axiologiques déclenchés et actualisés par les lecteurs. Dans tous les cas, le basculement de la lecture à la consommation de la marque apparaît fragile :

52 - L'arbitrage entre discrétion et présence de la marque peut conduire à la surexposer, au risque de décrédibiliser le message, ou à la masquer, au risque de promouvoir la catégorie de biens et services et non plus la marque en particulier.

53 - Ces magazines peuvent être pratiqués avec détachement et plus parcourus que lus.

54 - La conscience de la construction opérée autour des marques permet aux lecteurs de mettre le discours médiatique à distance et de «classer » cette tentative relationnelle dans le registre publicitaire.

- Leur acceptation de ce mode persuasif qualifié « d'intelligent » leur permet d'apprécier le processus publicitaire, sans pour autant avoir envie d'acheter auprès de l'enseigne éditrice; certains affirment d'ailleurs spontanément l'indépendance de la lecture effectuée et des choix marchands à venir.

Le caractère systématique qui impliquerait une attitude économique positive après l'exposition à des représentations, même séduisantes, n'est donc pas démontré (il ne s'agit pas évidemment de mettre en cause le pragmatisme mais de réaffirmer l'écart entre la finalité d'un message et l'absence de symétrie chez celui qui l'interprète). Comme l'a évoqué M. de Certeau, dans cette tentative de rapport de domination, le lecteur garde une certaine liberté qui lui permet de «bricoler » selon son désir. Le pouvoir d'autorité de la lecture est relativisé pour les magazines de marque par l'autonomie et «l'impertinence des lecteurs ». Leur parcours, chaotique et incertain, ne répond pas au parcours modèle et idéal-typique du contrat qui sous-tend les magazines de marque, entre lecture et consommation de la marque.

\section{Conclusion}

57 La relation proposée par les magazines semble désirable pour maximiser les échanges avec le lecteur et l'inscrire dans un système d'adhésion à la marque: rituelle, apparemment désintéressée, elle transforme le statut du discours de marque. La fidélité 
serait une notion contrôlable et accessible en fonction des ingrédients, formes et représentations intégrés dans un texte, sur lesquels les marques pourraient capitaliser pour faire adhérer à leur univers symbolique. Elle serait assujettie en particulier au respect d'un équilibre entre visibilité et discrétion de la marque dans le texte médiatique, entre vocation économique et communicationnelle. L'hybridité inhérente aux magazines de marque apparait ainsi à la fois comme la source de leur acceptation et le signe de leur instabilité relationnelle, marquée par les glissements.

Le terme de « contrat de lecture » repris par les professionnels des magazines de marque suggère l'engagement de la marque à respecter l'équilibre évoqué et l'engagement du lecteur à se laisser porter par le texte. Le recours au « contrat de lecture » souligne aussi le désir d'une systématisation de ce lien textuel et métaphorique d'un parcours glissant de la lecture à la consommation. En transformant le modèle du contrat en outil opératoire, la communication est envisagée comme un processus d'extension et de réduction des représentations, adaptables suivant les émetteurs, leurs objectifs et leurs destinataires, La sémiotique apparait ici comme un instrument de contrôle du sens permettant de concevoir les signes comme objets d'une gestion systématique: une gestion sémiotique apte à modéliser et structurer.

Pourtant l'acceptation d'un rendez-vous rituel avec la marque à travers ses magazines semble n'engager le lecteur que d'une façon discontinue et ne garantit pas un achat futur. Elle configure une fidélisation de papier, fondée sur le lien symbolique que chacun entretient avec les médias, la publicité, la consommation, qu'elle soit celle du magazine offert et/ou de la marque offrante.

La confrontation entre le modèle du contrat de lecture comme instrument de fidélisation et ce qu'il advient dans les mains des lecteurs souligne les coopérations mais aussi les divergences entre le travail de circonscription des cibles et la richesse de leurs propres représentations et pratiques.

\section{BIBLIOGRAPHIE}

Barthes R., L'aventure sémiologique, Paris, Seuil, Points, 1985.

Certeau (de) M., L'invention du quotidien, vol. 1, Art de faire, Paris, Gallimard, Folio, essais, 1990.

Cochoy F., Une histoire du marketing, Discipliner l'économie de marché, Paris, La découverte, 1999.

Floch J.M., Sémiotique, marketing et communication. Sous les signes les stratégies, Paris, PUF 1995 (1 ${ }^{\text {re }}$ édition : 1990).

Jeanneret Y. et Patrin-Leclère V., « La métaphore du contrat » in : Hermès 38, 2004. pp. 133-140.

Lévi-Strauss C., La pensée sauvage, Paris, Plon, Pocket, Agora, 1990 ( $1^{\text {re }}$ édition : 1962).

Marion G., « Le marketing relationnel existe-t-il ? » in : Décisions Marketing n²2. Janvier-avril 2001. pp. 7-16. 
Montety (de) C., « Magazines de marque : métamorphoses d'une promesse », Thèse en cours, Celsa Paris IV-Sorbonne.

Pasquier M., Marketing et sémiotique : une approche inter-disciplinaire, Fribourg, Editions Universitaires, 1999.

Semprini A., Le marketing de la marque, approche sémiotique, Paris, Liaisons, 1992.

Veron E., «L'analyse du contrat de lecture : une nouvelle méthode de positionnement des supports » in IREP, Médias, Paris, 1985, pp. 203-229.

\section{NOTES}

1. Corpus constitué des numéros diffusés tout au long de l'année 2002 de Room d'Ikea, Maisons en vie de Leroy Merlin, Mise en scène de Mobalpa et Truffaut Magazine.

2. Jeanneret Y., Patrin-Leclère V., «La métaphore du contrat» in: Hermès $n^{\circ} 38,2004$. pp. 133-140.

3. Ces types de valorisation sont souvent évoqués dans les études sur la consommation, par exemple par J.M. Floch et A. Semprini.

\section{RÉSUMÉS}

Cet article évoque la façon dont les pratiques du marketing relationnel, fortement naturalisées, font oublier les représentations sur lesquelles elles reposent. Ainsi, les magazines de marque semblent s'appuyer sur le métaphorique contrat de lecture pour proposer, à leurs cibles, une relation susceptible de les fidéliser. Cette construction d'une relation modélisée et systématisée se confronte toutefois à la déconstruction et à la manipulation des lecteurs. Cette fidélisation de papier, entre symboles et pratiques, apparaît fragile au regard de lecteurs et clients, libres de leur parcours de consommation.

This article proposes to show how the practises of relational marketing as largely "naturalized" induce profesionnals and targets to forget the representation on wich they are based. Brand magazines seem to be supported by a metaphorical reading contract to suggest a loyalty building relationship to their audiences. This build up of a modelised and systemised relationship is nevertheless confronted to deconstruction and readers manipulation. This "paper loyalty", between symbols and practises, seems fragile as readers and clients prove themselves free in their consumption journey.

\section{AUTEUR}

\section{CAROLINE DE MONTETY}

Caroline de Montety est doctorante en SIC au Celsa, ParisIV-Sorbonne et fait partie de l'équipe de recherche du GRIPIC. Elle s'intéresse particulièrement aux discours de marques. Mail :

caroline.demontety@free.fr 